\title{
燕山山脉黄檗种群结构与动态特征
}

\author{
王泳腾, 黄治昊, 王 俊, 张童, 崔国发* \\ 北京林业大学生态与自然保护学院,北京 100083
}

摘要:黄檗 (Phellodendron amurense) 为我国珍稀濒危物种, 具有较高的药用价值。以燕山地区野生黄檗种群为研究对象,采用典 型抽样对 8 个保护区内的黄檗进行全面调查, 通过编制静态生命表并绘制存活曲线, 分析种群年龄结构和种群变化规律, 并通 过种群动态量化和时间序列预测分析种群未来数量变化。结果表明: (1) 黄檗种群结构总体上呈倒“J”型,但存在外部干扰时, 种群结构数量变化动态指数为 $1.017 \%$, 表明该种群趋于稳定型; (2) 静态生命表及相关曲线分析显示, 黄檗种群存活数量整体 上随着径级增大, 个体数量逐渐下降; 黄檗种群的生存期望在不同径级间存在波动, 而第 VII径级的生存期望最高, 种群存活曲线 符合 Deevey- II 型,黄檗种群具有前期减少、中期稳定、后期衰退的特点; ( 3) 时间序列预测分析表明, 在未来 $2 、 4 、 6 、 8$ 个径级之 后, 黄檗中老龄个体数量将逐渐增加。黄檗种群濒危的主要因素是幼苗数量少, 且呈现散生分布状态, 建议加强幼苗抚育工作, 提高幼苗的存活率,保护和改善生境,从而促进黄檗种群的自然更新和恢复。

关键词:黄檗; 种群结构; 存活曲线; 时间序列预测

\section{The population structure and dynamic characteristics of Phellodendron amurense in Yanshan Mountains}

WANG Yongteng, HUANG Zhihao, WANG Jun,ZHANG Tong, CUI Guofa *

School of Ecology and Nature Conservation, Beijing Forestry University, Beijing 100083, China

\begin{abstract}
Amur corktree (Phellodendron amurense) is a rare and endangered species in China. This paper takes the wild Phellodendron amurense population in Yanshan Mountains as the research object. We conduct comprehensive survey on Phellodendron amurense in eight reserves through typical sampling, establish a static life table and draw a population survival curve to analyze the age structure of the population, and use its population dynamic quantization and time series analysis to predict potential population changes. The results show that (1) the $P$. amurense population is by an large of an inverted $\mathrm{J}$ type. The dynamic change index under external interference is $1.017 \%$, indicating that the population tends to be stable. (2) Analysis on the static life table and the population survival curve shows that the survival number of the $P$. amurense population increases along with the diameter scales as the individual number decreases, with the VII group at the top. The population survival rate belongs to the typical type of Deevey-II. The $P$. amurense population grows poorly in early age, stably in medium age period, and declines in old age. (3) Time series analysis indicates that, after more trees grow in the 2, 4, 6, 8 diameter scales in the future, the number of medium and old-aged trees in the population may increase gradually. The major factor for the endangering of the $P$. amurense population is its insufficient seedling regeneration and scattered tree distribution. Therefore, the paper concludes that seedling raising should be strengthened to increase the survival rate of the seedlings, and the living environment be protected and improved to enhance natural
\end{abstract}

基金项目:东部典型区域陆地生物多样性保护目标设定与评估示范(2018YFC0507203)

收稿日期: 2020-03-30; 网络出版日期:2021-01-27

* 通讯作者 Corresponding author.E-mail: fa6716@163.com 
regeneration and restoration of the $P$. amurense population.

Key Words: Phellodendron amurense; population structure; survival curve; time series analysis

种群生态学的核心是种群动态研究 ${ }^{[1-2]}$, 它涉及物种种群的动态以及这些种群如何与环境相互作用, 是 一项关于物种数量随时间和空间变化的研究 ${ }^{[3-4]}$ 。通过研究生物种群结构, 可以评估历史干扰, 并对种群未 来发展趋势进行预测 ${ }^{[5-6]}$ 。

黄檗 (Phellodendron amurense) 为芸香科 (Rutaceae) 黄檗属 (Phellodendron) 落叶乔木, 是古老的子遗植物。 由于黄檗具有抗炎、降血糖血压等重要的药用价值, 在 20 世纪末受到了严重的人为破坏, 野生种群数量日益 减少,世界自然保护联盟 (International Union for Conservation of Nature, IUCN) 将其列为易危 (Vulnerable, VU) 物种 ${ }^{[7]}$ 。目前我国黄檗研究主要集中在药用机理分析 ${ }^{[8-11]}$ 、人工栽培繁育 ${ }^{[12]}$ 、潜在适宜分布区预测 ${ }^{[13]}$ 等方 $^{-}$ 面。而对某一局部地区黄檗的种群生态学研究相对较少 ${ }^{[14-15]}$, 对燕山山脉这一地理尺度上黄檗种群的研究 还未见报道。为探究黄檗生态学特性,深人分析黄檗的受威胁状况、濒危程度, 阐明黄檗野生种群的濒危机 制 ${ }^{[16-17]}$, 为野生黄檗种群保护和利用提供科学理论依据 ${ }^{[18]}$, 需要对现存黄檗的种群结构和数量特征开展调查 与研究。

\section{1 材料和方法}

\section{1 研究区概况}

燕山位于东经 $115^{\circ}-119^{\circ} 47^{\prime}$, 北纬 $39^{\circ} 40^{\prime}-41^{\circ} 20^{\prime}$, 东西长约 $420 \mathrm{~km}$, 南北 $200 \mathrm{~km}$ 左右, 东猴顶为其最高 峰, 海拔 $2392 \mathrm{~m}$ 。燕山植被类型丰富, 既有蒙古栋( Quercus mongolica)、槲栋 ( Quercus aliena)、栓皮栋 ( Quercus variabilis)、槲树 (Quercus dentata)、白桦 (Betula platyphylla)、硕桦(Betula costata) 等落叶阔叶树, 又有臭冷杉 (Abies nephrolepis)、华北落叶松 (Larix principis-rupprechtii) 等针叶树种。燕山属暖温带大陆性季风气候区,夏 季炎热多雨, 冬季寒冷干燥。年均温 $6-10^{\circ} \mathrm{C}, 10^{\circ} \mathrm{C}$ 以上持续期 $195-205 \mathrm{~d}$, 活动积温 $2600-3800^{\circ} \mathrm{C}$, 降水季 节分配不均匀,全年降水的 $80 \%$ 集中在夏季 $6 、 7 、 8$ 三个月,年降水量 $700 \mathrm{~mm}$ 左右, 土地类型以棕壤、褐土 为主。

\section{2 调查方法}

由于黄檗分布稀少且在野外多呈现散生状态,在大量文献调查的基础上,于 2019 年 7-8 月,选择了燕山 范围内 8 个国家级自然保护区中黄檗分布较集中且自然林木保存完好的地点作为调查区域,对保护区内几乎 所有的黄檗都进行了详细样方调查 (图 1、表 1), 设置样地共计 33 块, 每个样地面积为 $20 \mathrm{~m} \times 20 \mathrm{~m}$, 对样地中的 乔木进行每木检尺,记录植物的名称、胸径、树高、冠幅、数量等等, 同时记录样地的海拔、经纬度、坡度、坡向、 坡位、郁闭度等生境条件。

\section{表 1 黄檗调查样地的基本概况}

Table 1 Basic Information of sample plots of Phellodendron amurense

\begin{tabular}{|c|c|c|c|c|c|}
\hline $\begin{array}{c}\text { 编号 } \\
\text { No. }\end{array}$ & $\begin{array}{l}\text { 调查地点 } \\
\text { Survey sites }\end{array}$ & $\begin{array}{c}\text { 坡位 } \\
\text { Slope positions }\end{array}$ & $\begin{array}{c}\text { 坡度 } \\
\text { Slope angles }\end{array}$ & $\begin{array}{c}\text { 坡向 } \\
\text { Directions }\end{array}$ & $\begin{array}{l}\text { 海拔/m } \\
\text { Altitudes }\end{array}$ \\
\hline 1 & 北京百花山国家级自然保护区 & 上坡位 & $17-22^{\circ}$ & $322^{\circ} \mathrm{NW}$ & 1295 \\
\hline 2 & & 上坡位 & $10-16^{\circ}$ & $328^{\circ} \mathrm{NW}$ & 1293 \\
\hline 3 & & 上坡位 & $3-8^{\circ}$ & $2^{\circ} \mathrm{N}$ & 1179 \\
\hline 4 & & 沟谷 & - & - & 1174 \\
\hline 5 & 北京松山国家级自然保护区 & 沟谷 & - & - & 962 \\
\hline 6 & & 上坡位 & $16-22^{\circ}$ & $56^{\circ} \mathrm{NE}$ & 1174 \\
\hline 7 & & 上坡位 & $15-21^{\circ}$ & $13^{\circ} \mathrm{W}$ & 906 \\
\hline 8 & 河北大海坨国家级自然保护区 & 下坡位 & $30-34^{\circ}$ & $30^{\circ} \mathrm{N}$ & 1008 \\
\hline
\end{tabular}




\begin{tabular}{|c|c|c|c|c|c|}
\hline \multicolumn{6}{|l|}{ 续表 } \\
\hline 编号 & 调查地点 & 坡位 & 坡度 & 坡向 & 海拔 $/ \mathrm{m}$ \\
\hline No. & Survey sites & Slope positions & Slope angles & Directions & Altitudes \\
\hline 9 & 河北茅荆坝国家级自然保护区 & 中坡位 & $13-19^{\circ}$ & $133^{\circ} \mathrm{SE}$ & 984 \\
\hline 10 & & 中坡位 & $3-7^{\circ}$ & $132^{\circ} \mathrm{SE}$ & 1081 \\
\hline 11 & & 中坡位 & $28-33^{\circ}$ & $3^{\circ} \mathrm{N}$ & 836 \\
\hline 12 & & 中坡位 & $8-13^{\circ}$ & $66^{\circ} \mathrm{NE}$ & 890 \\
\hline 13 & & 上坡位 & $12-17^{\circ}$ & $188^{\circ} \mathrm{S}$ & 1055 \\
\hline 14 & & 中坡位 & $7-12^{\circ}$ & $14^{\circ} \mathrm{N}$ & 837 \\
\hline 15 & 河北雾灵山国家级自然保护区 & 中坡位 & $23-28^{\circ}$ & $26^{\circ} \mathrm{NE}$ & 964 \\
\hline 16 & & 中坡位 & $18-23^{\circ}$ & $30^{\circ} \mathrm{NW}$ & 332 \\
\hline 17 & & 中坡位 & $11-16^{\circ}$ & $30^{\circ} \mathrm{NW}$ & 281 \\
\hline 18 & & 下坡位 & $23-28^{\circ}$ & $195^{\circ} \mathrm{S}$ & 410 \\
\hline 19 & & 下坡位 & $48-53^{\circ}$ & $17^{\circ} \mathrm{N}$ & 715 \\
\hline 20 & & 中坡位 & $24-29^{\circ}$ & $52^{\circ} \mathrm{NE}$ & 690 \\
\hline 21 & 辽宁白狼山国家级自然保护区 & 上坡位 & $3-8^{\circ}$ & $114^{\circ} \mathrm{SE}$ & 701 \\
\hline 22 & & 沟谷 & - & - & 707 \\
\hline 23 & & 中坡位 & $4-9^{\circ}$ & $133^{\circ} \mathrm{SE}$ & 671 \\
\hline 24 & & 沟谷 & - & - & 578 \\
\hline 25 & 辽宁虹螺山国家级自然保护区 & 沟谷 & - & - & 331 \\
\hline 26 & & 下坡位 & $17-22^{\circ}$ & $355^{\circ} \mathrm{N}$ & 341 \\
\hline 27 & & 下坡位 & $29-34^{\circ}$ & $332^{\circ} \mathrm{NW}$ & 413 \\
\hline 28 & & 下坡位 & $28-35^{\circ}$ & $9^{\circ} \mathrm{N}$ & 385 \\
\hline 29 & 天津八仙山国家级自然保护区 & 上坡位 & $33-39^{\circ}$ & $59^{\circ} \mathrm{NE}$ & 915 \\
\hline 30 & & 上坡位 & $38-43^{\circ}$ & $135^{\circ} \mathrm{SE}$ & 897 \\
\hline 31 & & 上坡位 & $18-23^{\circ}$ & $83^{\circ} \mathrm{E}$ & 910 \\
\hline 32 & & 上坡位 & $28-34^{\circ}$ & $355^{\circ} \mathrm{N}$ & 850 \\
\hline 33 & & 中坡位 & $17-22^{\circ}$ & $5^{\circ} \mathrm{N}$ & 535 \\
\hline
\end{tabular}

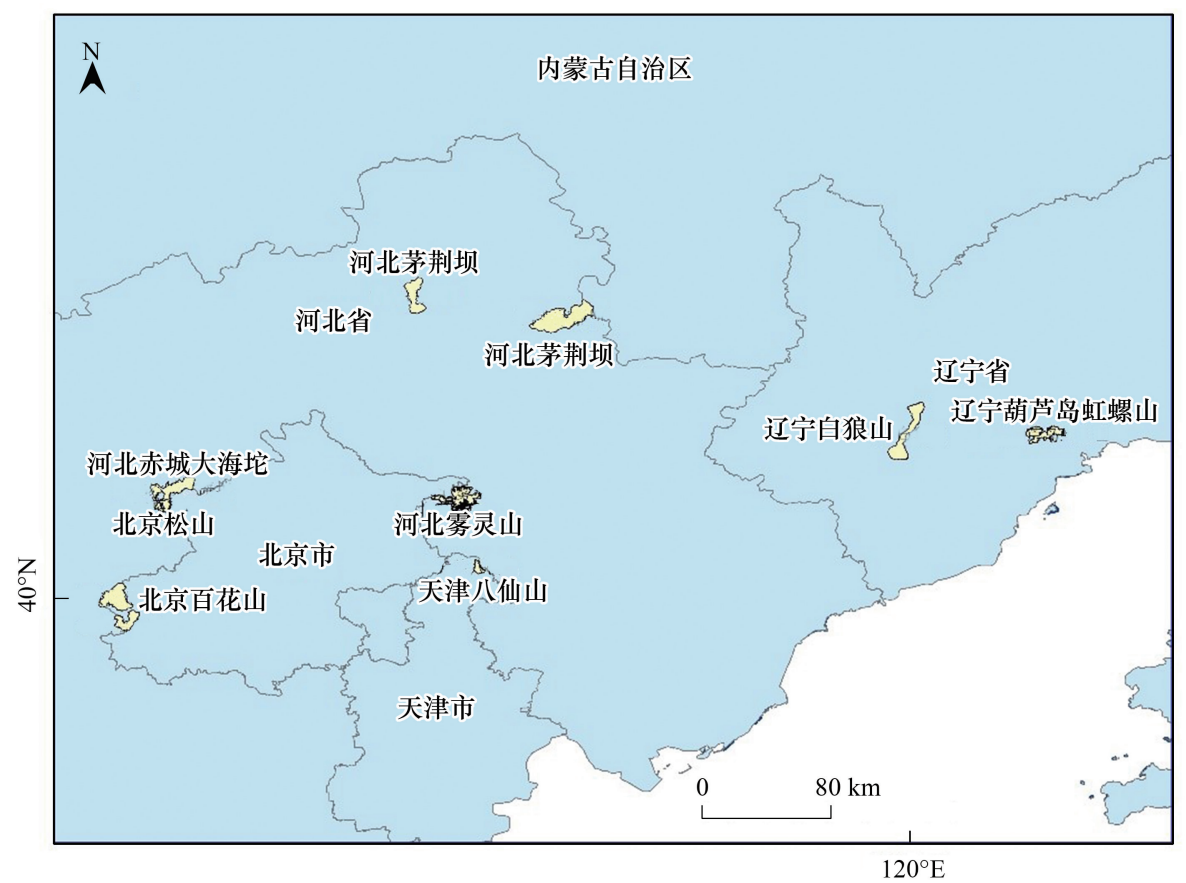

图 1 自然保护区位置分布图

Fig.1 Location map of Nature Reserves 


\section{3 数据分析}

\subsection{1 年龄结构划分}

黄檗作为濒危物种, 种群数量有限。选择径级结构代替年龄结构, 参考相关林木径级划分方法 ${ }^{[15][19]}$, 根 据黄檗的生长规律和生活史特征,将其划分为 11 个径级:第 I 径级为幼苗 $(\mathrm{DBH} \leqslant 4 \mathrm{~cm})$; 第 II 径级 $(4 \mathrm{~cm}<$ $\mathrm{DBH} \leqslant 8 \mathrm{~cm})$ 、第 III 径级 $(8 \mathrm{~cm}<\mathrm{DBH} \leqslant 12 \mathrm{~cm})$ 、第 $\mathrm{IV}$ 径级 $(12 \mathrm{~cm}<\mathrm{DBH} \leqslant 16 \mathrm{~cm})$ 为小树; 第 $\mathrm{V}$ 径级 $(16 \mathrm{~cm}<\mathrm{DBH} \leqslant$ $20 \mathrm{~cm})$ 、第 $\mathrm{VI}$ 径级 $(20 \mathrm{~cm}<\mathrm{DBH} \leqslant 24 \mathrm{~cm})$ 、第 VII径级 $(24 \mathrm{~cm}<\mathrm{DBH} \leqslant 28 \mathrm{~cm})$ 、第 VIII径级 $(28 \mathrm{~cm}<\mathrm{DBH} \leqslant 32 \mathrm{~cm})$ 为中 树; 第 $\mathrm{IX}$ 径级 $(32 \mathrm{~cm}<\mathrm{DBH} \leqslant 36 \mathrm{~cm})$ 、第 $\mathrm{X}$ 径级 $(36 \mathrm{~cm}<\mathrm{DBH} \leqslant 40 \mathrm{~cm})$ 、第 $\mathrm{XI}$ 径级 $(40 \mathrm{~cm}<\mathrm{DBH} \leqslant 44 \mathrm{~cm})$ 为大树 阶段。

\subsection{2 种群动态数量化方法}

参考陈晓德 ${ }^{[20]}$ 的量化方法,利用动态指数进行分析, 具体公式如下:

$$
\begin{aligned}
& v_{n}=\frac{S_{n}-S_{n+1}}{\max S_{n} S_{n+1}} \times 100 \% \\
& V_{p i}=\frac{\sum_{n=1}^{k=1}\left(S_{n} \times V_{n}\right)}{\sum_{n=1}^{k=1} S_{n}} \\
& V_{p i}^{\prime}=\frac{\sum_{n=1}^{k=1} S_{n} V_{n}}{k \times \min \left(S_{1}, S_{2}, \cdots S_{k}\right) \sum_{n=1}^{k=1} S_{n}} \\
& P=\frac{1}{k \times \min S_{1} S_{2} \cdots S_{k}}
\end{aligned}
$$

式中, $V_{n}$ 为从 $n$ 到 $n+1$ 级的个体数量变化; $V_{p i}$ 为不考虑未来外部环境干扰时, 整个种群结构的数量变化动态 指数; $V_{p i}^{\prime}$ 为考虑未来的外部干扰时, 整个种群结构的数量变化动态指数; $S_{n} 、 S_{n+1}$ 分别是第 $n$ 、第 $n+1$ 级的种群 个体数; $k$ 为种群大小级数量。

\subsection{3 种群静态生命表及存活曲线}

参考姜在民、赵阳 ${ }^{[21-22]}$ 的方法, 编制黄檗种群静态生命表, 由于静态生命表是根据某一特定时刻种群所 有个体的径级编制而成 ${ }^{[23]}$, 且此次调查中幼苗个体数量较少, 会出现死亡率为负的情况, 因此对 $A x$ 采用方程 拟合并做匀滑处理, 得到修正后的数据 $a_{x}$, 拟合时以径级为自变量, 存活数量为因变量, 得到的拟合方程为 $y=$ $182.06 \mathrm{e}^{-0.103 x}, R^{2}=0.8746$ 生命表主要包括以下参数,计算公式分别为:

$$
\begin{aligned}
& l_{x}=\left(a_{x} / a_{0}\right) \times 1000 ; \\
& d_{x}=l_{x}-l_{x+1} ; \\
& q_{x}=\left(d_{x} / l_{x}\right) \times 100 \% ; \\
& L_{x}=\left(l_{x}+l_{x+1}\right) / 2 ; \\
& T_{x}=L_{x}+L_{x+1}+L_{x+2} \cdots+L_{x+n} ; \\
& e_{x}=T_{x} / l_{x} ; \\
& K_{x}=\ln l_{x}-\ln l_{x+1} ; \\
& S_{x}=l_{x+1} / l_{x} ;
\end{aligned}
$$

式中, $l_{x}$ 为第 $x$ 径级的标准化存活个体数; $d_{x}$ 为从 $x$ 到 $x+1$ 径级间的标准化死亡数; $q_{x}$ 为第 $x$ 到 $x+1$ 径级间隔 期间死亡率; $L_{x}$ 为第 $x$ 到 $x+1$ 径级间隔期间仍存活的个体数; $T_{x}$ 为大于等于 $x$ 径级的存活个体总数; $e_{x}$ 为进人 第 $x$ 径级个体的生命期望或平均期望寿命; $K_{x}$ 为消失率; $S_{x}$ 为存活率。

此外, 依据 Helt 与 Loucks 的数学模型描述黄檗种群存活曲线, 即运用指数方程式 $N_{x}=N_{0} e^{-b x}$ 和幕函数 $N_{x}=N_{0} x^{-b}$ 分别描述 Deevey- II 型和 Deevey-III 型存活曲线 ${ }^{[24]}$ 。 
式中, $N_{x} 、 N_{0}$ 分别代 $x$ 径级内存活数和种群形成初期的个体数, $N_{x} 、 N_{0}$ 为经匀滑后的个体存活数, $b$ 为死亡率。

\subsection{4 生存分析曲线}

+为了更好地分析黄檗种群动态, 本文引人 4 个生存函数, 分别是种群生存函数 $S_{(i)}$ 、累计死亡率函数 $F_{(i)}$ 、死亡密度函数 $f_{(i)}$ 和危险率函数 $\lambda_{(i)}{ }^{[25-26]}$, 计算公式为:

式中, $S_{i}$ 为存活率; $h_{i}$ 为径级宽度。

$$
\begin{aligned}
& S_{(i)}=S_{1} \times S_{2} \times S_{3} \times \cdots \times S_{i} \\
& F_{(i)}=1-S_{(i)} \\
& f_{(i)}=\left(\mathrm{S}_{(i-1)}-\mathrm{S}_{(i)}\right) / h_{i} \\
& \lambda_{(i)}=2\left(1-S_{(i)}\right) /\left[h_{i}\left(1+S_{(i)}\right)\right]
\end{aligned}
$$

根据上述 4 个生存函数的估算值, 绘制生存率曲线、累计死亡率曲线、死亡密度曲线和危险率曲线。

1.3.5 种群数量动态的时间序列预测

采用一次移动平均法 ${ }^{[27]}$ 预测黄檗种群年龄结构, 其计算公式为:

$$
M_{t}=\frac{1}{n} \sum_{k=t-n+1}^{t} X_{k}
$$

式中, $t$ 为径级; $n$ 为需要预测的时间; $X_{k}$ 为当前 $k$ 径级的个体数量; $M_{t}$ 为 $t$ 径级黄檗在 $n$ 年之后的种群存活数。 本文 $t$ 分别取 $2 、 4 、 6 、 8$ 径级, 对种群数量进行预测。

\section{2 结果与分析}

2.1 黄檗种群分布现状及结构特征

本次调查到黄檗共计 334 株, 其中第 II 、III IV 径级个体数量较多, 分别占总数量的 $27.25 \%, 19.76 \% 、 15$. $57 \%$, 说明小树数量充足;第 I 径级个体数量占总数量的 $11.34 \%$, 可能是由于 21 世纪初期, 人类砍伐、盗伐现 象严重, 黄檗种群通过根蕃萌生, 由此产生一定数量的幼苗, 但由于更新能力不足, 导致数量不断减少; 第 X、 XI径级数量也较少, 仅占总数量的 $0.87 \%$, 说明黄檗种群老龄和幼龄个体数量匮乏; 第 II - VIII径级的种群数量 随径级的增大而减小。黄檗种群径级结构总体上呈倒“J”型(图 2), 为增长型种群。

根据表 2 可知, $V_{1}<0$, 说明第 I-II 径级生长过程中黄檗种群个体数量减少, 呈现衰退的结构动态; $V_{2} 、 V_{3}$ 、 $V_{4} 、 V_{5} 、 V_{6} 、 V_{7} 、 V_{9} 、 V_{10}$ 均大于 0 , 说明第 II - III III - IV 、 V $-\mathrm{V} 、 \mathrm{~V}-\mathrm{VI} 、 \mathrm{VI}-\mathrm{VII} 、 \mathrm{VII}-\mathrm{VII} 、 \mathrm{IX}-\mathrm{X} 、 \mathrm{X}-\mathrm{XI}$ 径级呈 现出增长的动态结构关系; $V_{8}=0$, 表明第 $\mathrm{VIII}-\mathrm{IX}$ 径级呈现稳定的结构动态关系, 种群总体上呈现增长趋势。 但种群年龄结构动态指数 $V_{p i}=20.333 \%$,大于存在外部干扰时的年龄结构动态变化指数 $V^{\prime}{ }_{p i}=1.017 \%$, 两者均 趋近于 $0, P_{\text {max }}=0.05$, 表明种群结构对随机干扰的敏感性指数为 0.05 , 说明黄檗种群抗干扰能力较低, 对环境 的敏感性较强, 虽有增长波动, 但趋于稳定型种群。

\section{表 2 黄檗种群动态变化指数}

Table 2 Dynamic index of Phellodendron amurense population

\begin{tabular}{ccc||ccc}
\hline $\begin{array}{c}\text { 径级 } \\
\text { Diameter scale }\end{array}$ & $\begin{array}{c}\text { 种群动态指数 } \\
\text { Dynamic index }\end{array}$ & $\begin{array}{c}\text { 动态指数值 } \\
\text { Dynamic index value/\% }\end{array}$ & $\begin{array}{c}\text { 径级 } \\
\text { Diameter scale }\end{array}$ & $\begin{array}{c}\text { 种群动态指数 } \\
\text { Dynamic index }\end{array}$ & $\begin{array}{c}\text { 动态指数值 } \\
\text { Dynamic index value/\% }\end{array}$ \\
\hline I & $V_{1}$ & -57.14 & VIII & $V_{8}$ & 0 \\
II & $V_{2}$ & 27.47 & IX & $V_{9}$ & 71.43 \\
III & $V_{3}$ & 21.21 & X & $V_{10}$ & 50 \\
IV & $V_{4}$ & 38.46 & XI & $V_{11}$ & 100 \\
V & $V_{5}$ & 25 & & $V_{p i}$ & \\
VI & $V_{6}$ & 45.83 & & $V_{p i}^{\prime}$ & 1.017 \\
II & $V_{7}$ & 46.15 & $P_{\text {max }}$ & & 0.05 \\
\hline
\end{tabular}




\section{2 黄檗种群静态生命表}

从表 3 和图 4 可知, 黄檗种群存活数量 $\left(l_{x}\right)$ 整体情 况为随着径级增大, 个体数量逐渐下降, 而死亡率 $\left(q_{x}\right)$ 和消失率 $\left(K_{x}\right)$ 在第 $\mathrm{V}$ 径级后波动浮动剧烈 (图 3 ), 说 明种群易受外界环境干扰, 种群状态不稳定。生命期望 $\left(e_{x}\right)$ 在第 $\mathrm{VI}$ 径级达到峰值, 说明此时黄檗正值生命力旺 盛的壮年时期,此后进人老龄时期,生理活动减弱,生命 期望值迅速降低,这种趋势符合生物学特征。

2.3 黄檗种群存活曲线

以径级 $(x)$ 为自变量, 以标准化存活数 $\left(l_{x}\right)$ 为因变 量, 绘制黄檗种群存活曲线 (图 4), 并拟合回归得到黄 檗种群相应存活曲线模型: $N_{x}=1309.2 \mathrm{e}^{-0.353 x}, R^{2}=$ $0.987, P<0.001$ 。

$$
N_{x}=1749.3 \mathrm{x}^{-1.514} \quad\left(R^{2}=0.915, P<0.001\right)
$$

结果表明, 指数函数模型的 $R^{2}$ 最大, 表明黄檗种群的存活曲线更趋近于各径级死亡率相似的 DeeveyII 型。

\section{表 3 黄檗种群静态生命表}

Table 3 Static life table of Phellodendron amurense population

\begin{tabular}{|c|c|c|c|c|c|c|c|c|c|c|c|c|}
\hline $\begin{array}{c}\text { 径级 } \\
\text { Diameter scale }\end{array}$ & $\begin{array}{c}\text { 胸径 } \\
\mathrm{DBH} / \mathrm{cm}\end{array}$ & $A x$ & $a_{x}$ & $l_{x}$ & $\ln l_{x}$ & $d_{x}$ & $q_{x}$ & $L_{x}$ & $T_{x}$ & $e_{x}$ & $K_{x}$ & $S_{x}$ \\
\hline I & $\leqslant 4$ & 39 & 121 & 1000.000 & 6.908 & 314.050 & 0.314 & 842.975 & 2607.438 & 2.607 & 0.377 & 0.686 \\
\hline II & $4-8$ & 91 & 83 & 685.950 & 6.531 & 223.140 & 0.325 & 574.380 & 1764.463 & 2.572 & 0.393 & 0.675 \\
\hline III & $8-12$ & 66 & 56 & 462.810 & 6.137 & 157.025 & 0.339 & 384.298 & 1190.083 & 2.571 & 0.414 & 0.661 \\
\hline IV & $12-16$ & 52 & 37 & 305.785 & 5.723 & 99.174 & 0.324 & 256.198 & 805.785 & 2.635 & 0.392 & 0.676 \\
\hline V & $16-20$ & 32 & 25 & 206.612 & 5.331 & 66.116 & 0.320 & 173.554 & 549.587 & 2.660 & 0.386 & 0.680 \\
\hline VI & $20-24$ & 24 & 17 & 140.496 & 4.945 & 49.587 & 0.353 & 115.702 & 376.033 & 2.676 & 0.435 & 0.647 \\
\hline VII & $24-28$ & 13 & 11 & 90.909 & 4.510 & 16.529 & 0.182 & 82.645 & 260.331 & 2.864 & 0.201 & 0.818 \\
\hline VIII & $28-32$ & 7 & 9 & 74.380 & 4.309 & 0.000 & 0.000 & 74.380 & 177.686 & 2.389 & 0.000 & 1.000 \\
\hline IX & $32-36$ & 7 & 9 & 74.380 & 4.309 & 33.058 & 0.444 & 57.851 & 103.306 & 1.389 & 0.588 & 0.556 \\
\hline $\mathrm{X}$ & $36-40$ & 2 & 5 & 41.322 & 3.721 & 16.529 & 0.400 & 33.058 & 45.455 & 1.100 & 0.511 & 0.600 \\
\hline XI & $40-44$ & 1 & 3 & 24.793 & 3.211 & 24.793 & 1.000 & 12.397 & 12.397 & 0.500 & 3.211 & 0.000 \\
\hline
\end{tabular}

$A x$ :存活数 individual number of diameter scale $x ; a_{x}$ : 修正后存活数 the revised data of $A x ; l_{x}$ : 第 $x$ 径级的标准化存活数 the standardized number of surviving individuals of diameter scale $x ; \ln l_{x}$ : 标准化存活数的自然对数 the natural logarithm of $l_{x} ; d_{x}$ : 从 $x$ 到 $x+1$ 径级间的标准化死亡数 the standardized number of death individuals from diameter scale $x$ to diameter scale $x+1 ; q_{x}$ : 第 $x$ 到 $x+1$ 径级间隔期间死亡率 mortality from diameter scale $x$ to diameter scale $x+1 ; L_{x}$ :第 $x$ 到 $x+1$ 径级间隔 期间仍存活的个体数 the number of surviving individuals from diameter scale $x$ to diameter scale $x+1 ; T_{x}$ : 大于等于 $x$ 径级的存活个体总数 the total individuals number of diameter scale $x$ and diameter scale elder than $x ; e_{x}$ : 进人第 $x$ 径级个体的生命期望或平均期望寿命 life expectancy or average life expectancy of individuals in the diameter scale $x ; K_{x}$ : 消失率 Vanish rate; $S_{x}$ :存活率 Survival rate

\section{4 黄檗种群生存变化规律}

黄檗种群累计死亡率 $F_{(i)}$ 随径级增大而上升,生存率 $S_{(i)}$ 随径级增大而下降,两者趋势相反。第 $\mathrm{I}-\mathrm{VI}$ 径 级之间,两者升降变化剧烈, 说明黄檗幼龄种群存活率低, 自然更新受阻。在第 VI径级之后,两条曲线的变化 趋于缓和(图 5)。

黄檗种群危险率函数 $\lambda_{(i)}$ 整体呈单调递增, 死亡密度函数 $f_{(i)}$ 在第 $\mathrm{I}-\mathrm{II}$ 径级间急剧下降 $($ 图 6$)$, 在第 VIII 径级出现最小值 $(0)$, 这说明黄檗种群开始进人生理衰老期, 种群开始衰退。4 个生存函数曲线表明, 黄檗种 
群动态表现为前期减少、中期稳定、后期衰退。

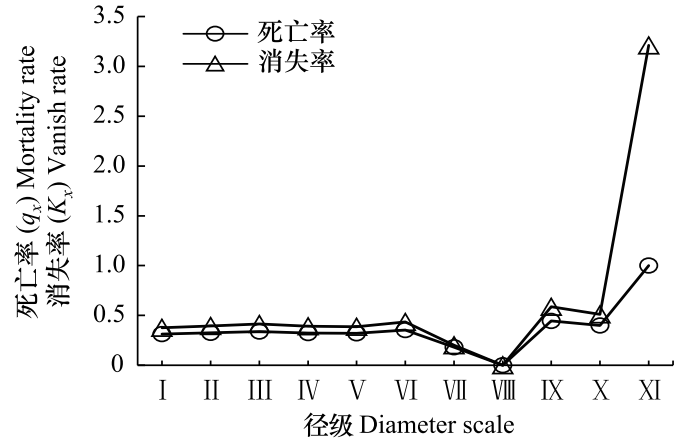

图 3 黄檗种群死亡率 $\boldsymbol{q}_{x}$ 和消失率 $\boldsymbol{K}_{x}$ 曲线

Fig. 3 Mortality $q_{x}$ and killing $K_{x}$ curves of Phellodend ron

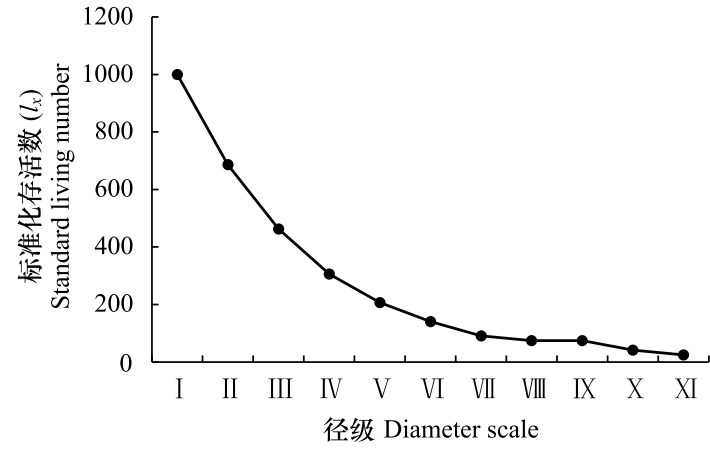

图 4 黄檗种群标准化存活曲线

Fig.4 Survival curve of Phellodendron amurense amurense

表 4 黄檗种群的生存函数估算值

Table 4 Survival functional rate of Phellodendron amurense population

\begin{tabular}{|c|c|c|c|c|}
\hline 径级 Diameter scale & $S_{(i)}$ & $F_{(i)}$ & $f_{(i)}$ & $\lambda_{(i)}$ \\
\hline I & 0.6860 & 0.3140 & 0.1715 & 0.6281 \\
\hline II & 0.4628 & 0.5372 & 0.0558 & 1.0744 \\
\hline III & 0.3058 & 0.6942 & 0.0393 & 1.3884 \\
\hline IV & 0.2066 & 0.7934 & 0.0248 & 1.5868 \\
\hline V & 0.1405 & 0.8595 & 0.0165 & 1.7190 \\
\hline VI & 0.0909 & 0.9091 & 0.0124 & 1.8182 \\
\hline VII & 0.0744 & 0.9256 & 0.0041 & 1.8512 \\
\hline VIII & 0.0744 & 0.9256 & 0.0000 & 1.8512 \\
\hline IX & 0.0413 & 0.9587 & 0.0083 & 1.9174 \\
\hline $\mathrm{X}$ & 0.0248 & 0.9752 & 0.0041 & 1.9504 \\
\hline XI & 0.0000 & 1.0000 & 0.0062 & 2.0000 \\
\hline
\end{tabular}

$S_{(i)}$ : 生存率 Survival rate; $F_{(i)}$ : 累计死亡率 Comulative morality rate; $f_{(i)}$ : 死亡密度 Mortality density; $\lambda(i)$ : 危险率 Hazard rate

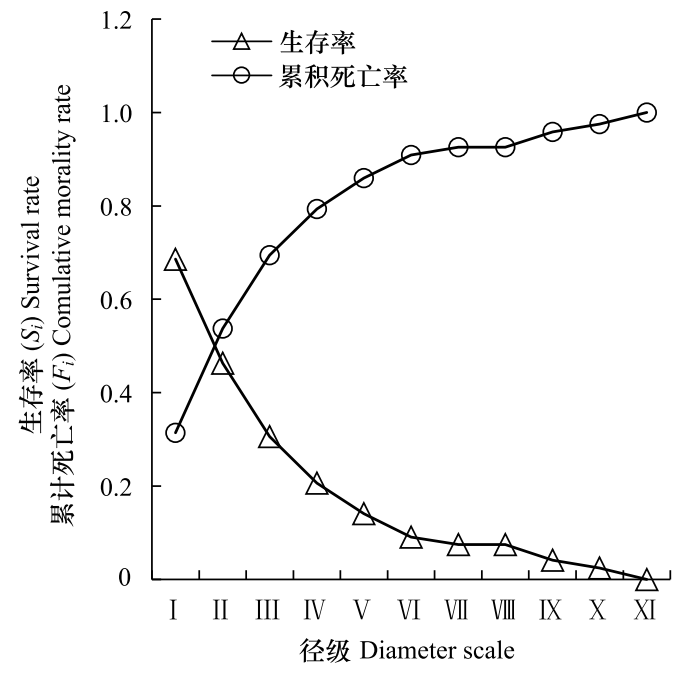

图 5 黄檗种群生存率 $S_{(i)}$ 和累积死亡率 $\boldsymbol{F}_{(i)}$ 曲线

Fig.5 Survival Rate $S_{(i)}$ and Comulative Mortality Rate $F_{(i)}$

Funcional Curve of Phellodendron amurense

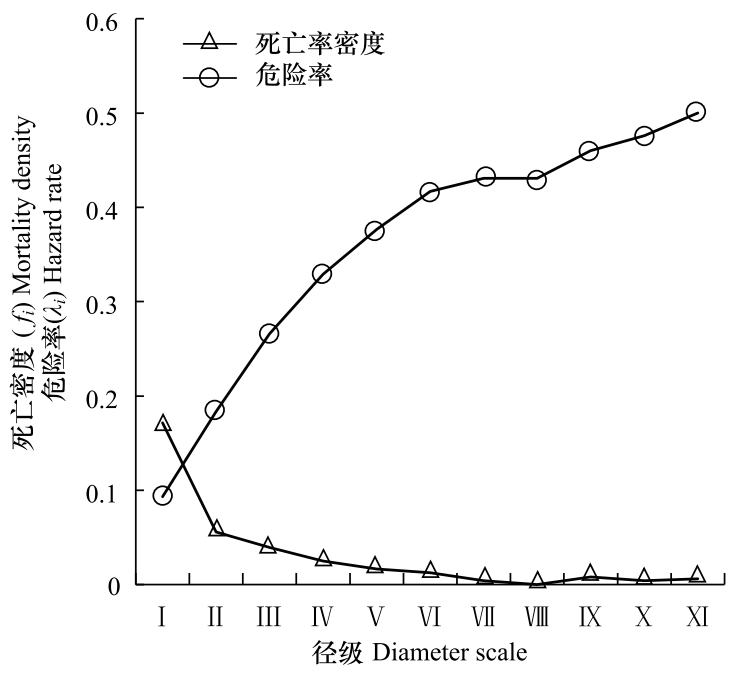

图 6 黄檗种群死亡密度 $f_{(i)}$ 和危险率 $\lambda_{(i)}$ 曲线

Fig. 6 Mortality Density $f_{(i)}$ and Hazard Rate $\lambda_{(i)}$ Funcional Curve of Phellodendron amurense 


\section{5 黄檗种群时间序列预测}

以黄檗种群各径级株数为原始数据, 按照一次移动平均法预测各径级在未来 $2 、 4 、 6 、 8$ 个径级时间后的个 体数(表 6、图 7)。经历 2 个径级时间后,第 II 径级的数量减少,表明黄檗幼苗数量少, 自我更新能力较差; 其 他径级在 $2 、 4 、 6 、 8$ 个径级之后的数量均有不同幅度的增加, 表明黄檗种群的增长趋势主要依靠中年和老年树 木,在外界环境保持良好的前提下,黄檗种群会呈现增长趋势。

表 6 黄檗种群数量动态时间序列预测

Table 6 Time sequence predivtion of Phellodendron amurense population

\begin{tabular}{|c|c|c|c|c|c|}
\hline $\begin{array}{c}\text { 径级 } \\
\text { Diameter scale }\end{array}$ & $\begin{array}{c}\text { 原始数据 } \\
\text { Primary data }\end{array}$ & $\mathrm{M}_{2}{ }^{(1)}$ & $\mathrm{M}_{4}{ }^{(1)}$ & $\mathrm{M}_{6}{ }^{(1)}$ & $\mathrm{M}_{8}{ }^{(1)}$ \\
\hline I & 39 & & & & \\
\hline II & 91 & 65 & & & \\
\hline III & 66 & 79 & & & \\
\hline IV & 52 & 59 & 62 & & \\
\hline $\mathrm{V}$ & 32 & 42 & 60 & & \\
\hline VI & 24 & 28 & 44 & 51 & \\
\hline VII & 13 & 19 & 30 & 46 & \\
\hline VIII & 7 & 10 & 19 & 32 & 41 \\
\hline IX & 7 & 7 & 13 & 23 & 37 \\
\hline X & 2 & 5 & 7 & 14 & 25 \\
\hline XI & 1 & 2 & 4 & 9 & 17 \\
\hline
\end{tabular}

$M_{t}{ }^{(1)}$ : 经过 2、4、6 和 8 径级时间后 $\mathrm{t}$ 径级的种群大小 the population size of diameter scale $\mathrm{t}$ after the time of $2,4,6,8$ diameter scale; (1) : 移动 平均法中的一次移动平均法标识 the mark of one-time moving average in moving average method

\section{3 结论和讨论}

燕山地区野生黄檗种群幼苗数量较少,低龄树木和 中龄树木数量较多,推测这可能与人类砍伐、盗伐有关, 被砍伐后的黄檗开始出现一定数量的幼苗萌蓝,但现存 小树 (第 II - IV 径级) 数目较多, 伴随着树木生长, 个体 对水分、光照、空间等资源需求增加,种内和种间竞争加 剧, 使得小树部分死亡, 具有较强生存力的中龄个体得 以存活,数量减少的趋势变缓, 种群趋于稳定状态, 黄檗 种群径级结构总体上呈倒 “ J”型, 是增长型种群, 这与 宋超 ${ }^{99}$ 等对北京地区黄檗种群的研究结果相似。但 $V_{p i}^{\prime}=1.017$ 趋近于 0 ,表明黄檗种群对外界的抗干扰能 力较弱,在生长过程中具有一定的波动性,但由于低龄 树和中龄树木较充分, 可以保持其稳定状态。

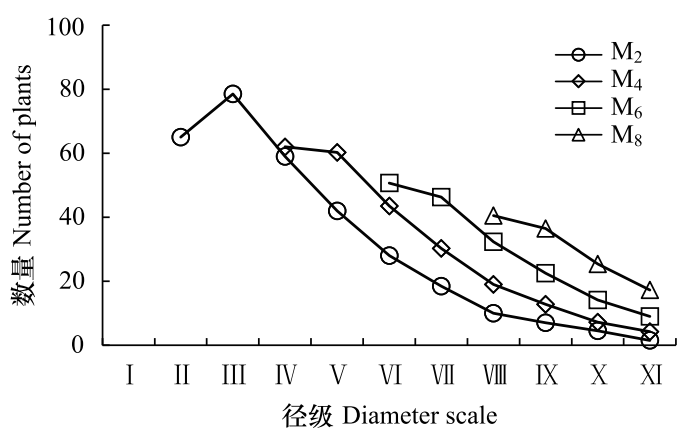

图 7 黄檗种群数量动态的时间序列预测

Fig.7 Time sequence predivtion of Phellodendron amurense population

$M_{t}{ }^{(1)}$ : 经过 2、4、6 和 8 径级时间后 $\mathrm{t}$ 径级的种群大小 the population size of diameter scale $t$ after the time of $2,4,6,8$ diameter scale

本研究黄檗种群的存活曲线为 Deevey- II 型, 这与 张强 ${ }^{[28]}$ 对辽宁省黄檗种群特征研究中黄檗存活曲线类型一致,但与易雪梅等 ${ }^{[8]}$ 对长白山地区黄檗种群存活 曲线为 Deevey-III不一致, 推测这与长白山地区红松、白桦等针阔叶树的数量居多, 且树高冠密, 较强地抑制了 黄檗幼苗的生长有关。种群死亡率和消失率曲线变化基本一致,都呈现先减少后增加的趋势。黄檗种群前 4 个径级的累积死亡率增长趋势显著高于后 7 个径级,这可能是由于黄檗幼龄和低龄个体在生长发育过程中对 光照、水分等资源的竞争力不足, 说明此时是造成黄檗种群生长波动的重要时期。植物种群的发展不仅涉及 到种内种间竞争,种群与环境之间的相互适应过程对其发展也有着重要影响 ${ }^{[29]}$ 。生存期望表现为先下降后 上升再下降的趋势, 推测黄檗种群在中龄时对环境的适应和生存能力最强, 而进入衰老期, 个体对资源不能进 
行充分利用,数量减少,种群衰退。这与其种群前期减少、中期稳定、后期衰退的结构特征相一致。

黄檗具有一定的萌蓝能力, 种子繁殖也是其种群更新的方式之一。燕山地区黄檗种群低龄数量丰富, 年 龄结构相对完整,短期内不会出现毁灭性的衰退 ${ }^{[30]}$ 。但是,在本次调查的某些保护区内,仍存在黄檗的盗伐 (剥皮)现象,将砍伐后的黄檗树干用来制作枕头治疗颈椎。目前我国采取就地保护、迁地保护、回归自然等 措施开展珍稀濒危野生植物保护,鉴于黄檗当前现状,建议重点保护和改善现存黄檗周围环境种群的生境条 件,一定程度上限制人类进人黄檗生境区域, 从而降低人为破坏; 其次, 黄檗为阳性树种, 对光照要求较高, 因 此可进行适度的人工修枝, 对一些竞争力较强的树种, 如胡桃楸 (Juglans mandshurica)、大果榆 (Ulmus macrocarpa) 进行修枝或者择伐, 改善林内光照 ${ }^{[31]}$; 此外, 黄檗幼苗数量不够乐观, 应及时清除幼苗周围的杂 草、灌木等, 为幼苗生长提供充足的空间和资源, 提高幼苗的存活率, 使更小的植株能够进入林冠层, 提高中、 成年树的比例。

本文从燕山这一大区域来对黄檗进行了整体研究和分析,但由于每个保护区存在生境异质性,不同保护 区内黄檗所处的群落也未尽相同,而海拔、坡度、坡向等也是影响黄檗群落动态的主要因素,下一步将开展影 响黄檗种群生长的微生境因子研究, 探讨其濒危机制和致危因素, 为黄檗种群的快速恢复和保育提供重要的 理论依据。

\section{参考文献 (References) :}

[1 ] 杨彪, 张全建, 龚旭, 段晨松, 张远涁. 雅聋江冬麻豆 (Salweenia bouffordiana) 种群结构与动态特征. 生态学报, 2020, 40(4)：1184-1194.

[ 2 ] 张志祥, 刘鹏, 蔡妙珍, 康华靖, 廖承川, 刘春生, 楼中华. 九龙山珍稀濒危植物南方铁杉种群数量动态. 植物生态学报, 2008, 32( 5): 1146-1156.

[ 3 ] Omelko A, Ukhvatkina O, Zhmerenetsky A, Sibirina L, Petrenko T, Bobrovsky M. From young to adult trees: how spatial patterns of plants with different life strategies change during age development in an old-growth Korean pine-broadleaved forest. Forest Ecology and Management, 2018, 411: 46-66.

［4］吉也, 曹孟岩, 白楚锋, 金林琦, 李畅, 李萍. 峨眉山杪椤种群结构与动态特征. 西北植物学报, 2019, 39(3): 543-551.

[ 5 ] 朱贵珍, 丁彦芬, 卓启苗, 余慧, 董丽娜. 南京紫金山红柴枝种群结构及动态特征. 东北林业大学学报, 2020, 48(1): 29-33.

[ 6 ] Harper J L. Population Biology of Plants. London: Academic Press, 1977.

[ 7 ] IUCN. IUCN Red List Categories and Criteria, Version 3.1. Switzerland and Cambridge, UK: IUCN, 2001.

[8] 秦彦杰. 黄檗主要药用成分的分布规律研究 [D]. 哈尔滨: 东北林业大学, 2005.

[9] 祖元刚, 王延兵, 王慧梅, 孙莲慧. 黄檗 (Phellodendron amuranse) 叶片总 RNA 提取方法研究. 植物研究, 2007, 27 (5) : 593-595.

[10］高杨，刘铭，张洪文. 珍稀植物黄檗的药用价值及可持续利用. 中国保健营养，2012，22(3): 22-24

[11] Lis A, Boczek E, Góra J. Chemical composition of the essential oils from fruits, leaves and flowers of the Amur cork tree (Phellodendron amurense Rupr. ). Flavour and Fragrance Journal, 2004, 19(6): 549-553.

[12] 刘琰璐, 戴灵超, 张昭. 黄檗繁殖技术研究进展. 中央民族大学学报: 自然科学版, 2011, 20(2): 84-87.

［13］黄治吴, 周金金, 张孝然, 蒲真, 邢韶华. 我国大陆黄檗潜在分布区及分布适宜性评价. 生态学报, 2018, 38(20) : 7469-7476.

［14］易雪梅, 张悦, 姬兰柱. 长白山黄檗种群结构.生态学杂志, 2013, 32(9): 2257-2262.

[15] 宋超, 余琦殷, 于梦凡, 邢韶华, 刘润泽, 曲宏, 黄三祥. 北京地区黄檗种群数量结构及空间分布特征. 生态学杂志, 2015, 34(11)： 3040-3049.

[16] Rozas V. Structural heterogeneity and tree spatial patterns in an old-growth deciduous lowland forest in Cantabria, northern Spain. Plant Ecology, 2006, 185(1): 57-72.

［17］秦彦杰，王洋，阎秀峰. 中国黄檗资源现状及可持续利用对策. 中草药, 2006, 37(7) : 1104-1107.

[18] 李翔, 侯璐, 李双喜, 李颖岳. 濒危树种庙台槭种群数量特征及动态分析. 植物科学学报, 2018, 36(4): 524-533.

[19] 何亚平, 费世民, 蒋俊明, 陈秀明, 张旭东, 何飞. 不同龄级划分方法对种群存活分析的影响一一水灾迹地油松和华山松种群生存分 析为例. 植物生态学报, $2008,32(2): 448-455$.

[20］陈晓德. 植物种群与群落结构动态量化分析方法研究. 生态学报, 1998, 18(2): 214-217.

[21］姜在民, 和子森, 宿吴, 赵涵, 蔡靖. 濒危植物羽叶丁香种群结构与动态特征. 生态学报, 2018, 38(7): 2471-2480.

[22] 赵阳, 齐瑞, 焦健, 陈学龙, 曹家豪, 冯宜明, 杨萌萌. 尔海-则岔地区紫果云杉种群结构与动态特征. 生态学报, 2018, 38(20): 7447-7457.

[23] 李俊清. 森林生态学. 北京: 高等教育出版社, 2006.

[24] Hett J M, Loucks O L. Age structure models of balsam fir and eastern hemlock. Journal of Ecology, 1976, 64(3) : 1029-1044.

[25] 冯士雍. 生存分析 ( I ). 数学的实践与认识, 1982, (3) : 72-80.

[26] 杨风翔, 王顺庆, 徐海根, 李邦庆. 生存分析理论及其在研究生命表中的应用. 生态学报, 1991, 11(2): 153-158.

[27] 王飞, 霍怀成, 赵阳, 曹秀文, 刘锦乾, 杨永红, 张涛, 齐吴, 王若鉴. 甘南高山林线岷江冷杉一一杜鹃种群结构与动态. 植物研究, 2019, 39(5): 664-672.

[28］张强. 辽宁省黄檗种群特征研究. 防护林科技, 2019, (1): 21-22.

[29] 王进, 姚兰, 艾训儒, 朱江, 刘松柏. 鄂西南不同区域亮叶桦种群结构与动态特征. 应用生态学报, 2020, 31(2): 357-365.

[30] 肖书礼, 付梦媛, 杨科, 陈小红. 极小种群野生植物峨眉含笑的种群结构与数量动态. 西北植物学报, 2019, 39(7): 1279-1288.

[31] 朱强, 艾训儒, 姚兰, 朱江, 吴漫玲, 黄小, 王进, 洪建峰. 鄂西南川陕鹅耳杵种群结构与动态. 中南林业科技大学学报, 2019, 39(8): 93-100. 